\title{
Technology for transformation of high-viscosity and high-paraffin oil raw materials using electromagnetic processing
}

\author{
N.K. Zhakirova ${ }^{\mathrm{a}}$, R.Kh. Salakhov ${ }^{\mathrm{b}}$, Zh.K. Nasim ${ }^{\mathrm{a}}$, A.M. Bakyt ${ }^{\mathrm{a}}$, L.R. Sassykova ${ }^{\mathrm{a}}$,

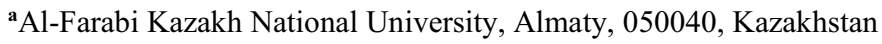 \\ b RSE “Institute for Combustion Problems”, Almaty, Kazakhstan
}

\begin{abstract}
The article presents the results of electromagnetic activation of petroleum raw materials in the vortex layer apparatus. It is shown that under electromagnetic influence, there is a significant increase in the proportion of straight-run gasoline fraction distillate, as well as a change in the physicochemical parameters of the light fractions obtained. It is established that the processes of wave action on oil, which take place in the zone of the electromagnetic field, lead to a change in the group hydrocarbon composition of the distillates obtained. NMR spectroscopy was used to study the effect of electromagnetic radiation on the properties of oil raw materials on the physicochemical properties and structure of the oil.
\end{abstract}

\section{Introduction}

The oil and gas industry will have to pay more and more attention to the development and commissioning of heavy, high-viscosity, hard-to-recover oil fields. The extraction, preparation and transportation of such oils is often complicated and sometimes impossible due to its low mobility due to the high viscosity of this oil. The field of scientific activity during the introduction of heavy high-viscosity oils into development is expanding. In the world, light oils are extracted no more than $50 \%$, heavy high-viscosity oils in the range of 10 to $30 \%$, depending on the characteristics of oil, water and reservoir [1-2].

At present, scientific developments have been intensified using non-traditional methods of activating oil, oil residues and bottom sediments.

Activation of petroleum raw materials by wave, including electromagnetic processing, for example, before the stage of primary atmospheric-vacuum distillation is one of the extraordinary ways to extract and increase light and medium distillate fractions beyond their potential content in oil and cubic heavy oil residues of secondary processes of advanced processing. At the same time, many physical and chemical properties, operational characteristics and technical and economic indicators of the resulting petroleum products are improved. It is known that the activation of petroleum raw materials is accompanied by

${ }^{1}$ nasimzhanerke@gmail.com 
a qualitative and quantitative change in the fractional, chemical, elemental and group compositions of light distillates obtained under atmospheric conditions and fractions during vacuum distillation of heavy residual oil products [3]. It is shown that under wave effects, reactions of moderate cracking of high-molecular hydrocarbons of mixed structure and polycondensed components of hydrocarbon raw materials occur under milder conditions than thermal cracking or visbreaking processes, which leads to changes in the hydrocarbon, fractional and component compositions of the distilled fractions [4].

In this paper, experimental studies on the electromagnetic activation of high-viscosity and high-paraffin oil samples are studied.

\section{Experimental part}

It is known that any change in the rheological and physico-chemical properties of oil dispersed systems (ODS) is accompanied by the transmission of energy to the system through thermal, mechanical, electronic, electromagnetic, acoustic, cavitation, radiation, and chemical effects. The evolution of the structural and chemical states, the stability of ODS, depends on the type of impact, the device through which the energy is transmitted, the time of energy transfer, and the amount of energy communicated to the medium. Practical intensification of the processes of field oil preparation and improvement of the efficiency of its processing with the use of electromagnetic processing the main attention is paid to the development of a laboratory apparatus with a vortex layer (AVL) and experimental studies on the electromagnetic activation of high-viscosity and high-paraffin oil samples. The device is capable of emitting a rotating electromagnetic field with a frequency of up to $300 \mathrm{~Hz}$ in the presence of ferromagnets. The initial oil feedstock in the tank, together with ferromagnets made in the form of needles, was placed in the working chamber of the device with a vortex layer. During the operation of the device, not the rotational motion of ferromagnetic particles is observed, but the Brownian motion resulting from repeated magnetization.

As a result of experimental studies during processing under static operating conditions of the laboratory AVL, we came to determine the efficiency of low-temperature cracking of hydrocarbon raw materials, improving the low-temperature properties and rheological parameters of activated oil, increasing the selection of light distillates in excess of their potential content in the feedstock, and improving the performance characteristics of the distillates obtained after atmospheric distillation of activated petroleum raw materials.

\section{Results and discussion}

The results of studies of the properties of oil and the fractional composition of its light part showed that the oil is sulfurous and contains a small amount of light distillates, namely: the gasoline fraction $\left(200^{\circ} \mathrm{C}\right)-14.97 \%$ by wt., the average distillate fraction $\left(200-300^{\circ} \mathrm{C}\right)$ $17.37 \%$ by wt. (Table 1 ). The results obtained in the study of the group compositions of gasoline fractions (Table 2) indicate that under electromagnetic influence on oil raw materials, as a result of the reactions of naphthene dehydrogenation and isoparaffin dehydrocyclization in a vortex magnetic activator, medium-low-temperature cracking occurs, which is accompanied by the destruction of the active intermolecular structures of the high-molecular part of the oil dispersed system and the chemical transformation of relatively low-molecular hydrocarbons, the formation of preferred mono-isomer compounds. 
Table 1. Physico-chemical characteristics of the oil studied.

\begin{tabular}{|l|c|}
\hline \multicolumn{1}{|c|}{ Indicator } & Value \\
\hline Relative density $\left(\rho^{20}{ }^{4}\right):$ & 0.890 \\
\hline Kinematic viscosity, $\mathrm{mm}^{2} / \mathrm{s}:$ & \\
at $20^{\circ} \mathrm{C}$ & 22.77 \\
at $60^{\circ} \mathrm{C}$ & 11.68 \\
\hline The water content, \% by wt. & traces \\
\hline The sulfur content, \% by wt. & 3.56 \\
\hline The content of surfactants, \% by wt. & 15.46 \\
\hline The yield of fractions, by wt.: & 14.97 \\
initial boiling point $-200{ }^{\circ} \mathrm{C}$ & 17.37 \\
\hline $200-300^{\circ} \mathrm{C}$ & \\
\hline
\end{tabular}

Due to the fluctuation, the productivity of aromatic hydrocarbons, in general, and the gasoline fraction increases. It is accompanied by the transition of polycyclic aromatic hydrocarbons and their complex structural unit (CSU), obtained from the adsorption-salt layer of the adsorption solvent in the extreme state, to the oil-loading dispersion system. This leads to the redistribution of hydrocarbons between phases and fractions, the transition from high-boiling oil fractions to low-boiling ones. To confirm the hypothetical assumptions, experimental studies were conducted to identify possible changes in the quantitative composition of resinous-asphaltene substances (RAS) in oil before and after electromagnetic treatment.

A decrease in the number of RAS during activation was found. This fully corresponds to the data given in Table 1. In particular, the content of aromatic hydrocarbons in activated oil increases from $14.1 \%$ to $21.7 \%$ during a 3-minute electromagnetic exposure. Compared to the content of cyclanes in the feedstock, only $50 \%$ of the chemical transformation occurs as a result of the naphthene dehydrogenation reaction. At the same time, the naphthene content in the activated oil will decrease only by $2 \%$, that is, from 20.7 to $18.8 \%$. Consequently, the rest of the chemical transformations associated with the increase in arenes are associated with the transition of complex structural units to the active extreme state, and then with their decay and the phase transition of intermolecular structures.

Table 2. Group composition of straight-run gasoline fractions obtained before and after activation.

\begin{tabular}{|c|c|c|c|}
\hline $\begin{array}{c}\text { Group } \\
\text { composition }\end{array}$ & $\begin{array}{c}\text { Without } \\
\text { activation }\end{array}$ & $\begin{array}{c}\text { Time of activation 3 } \\
\text { min. }\end{array}$ & $\begin{array}{c}\text { Time of activation 5 } \\
\text { min. }\end{array}$ \\
\hline paraffins & 29.455 & 30.149 & 29.842 \\
\hline isomers & 35.754 & 29.335 & 32.219 \\
\hline aromatics & 14.113 & 21.666 & 20.108 \\
\hline naphthenes & 20.669 & 18.787 & 17.759 \\
\hline olefins & 0.027 & 0.057 & 0.06 \\
\hline
\end{tabular}




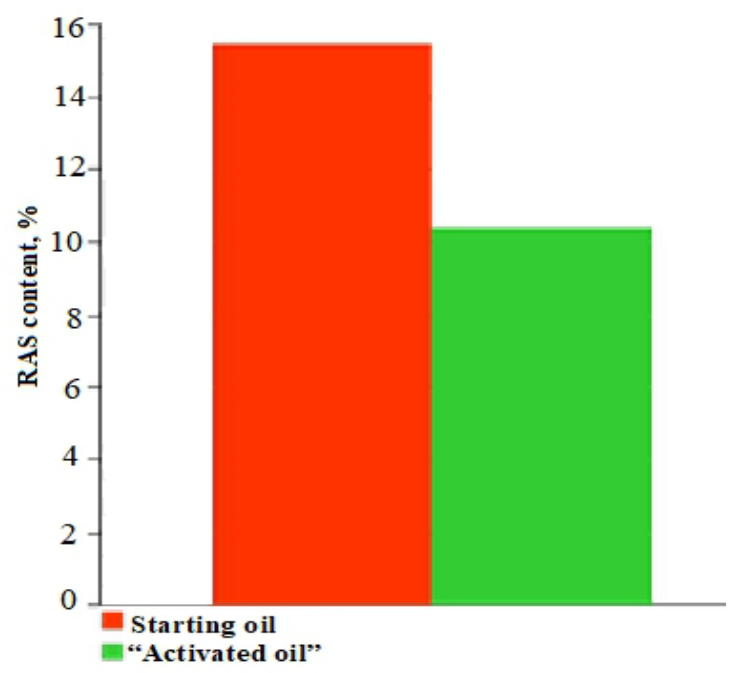

Fig. 1. The content of RAS in oil before and after electromagnetic treatment.

Any chemical transformations of petroleum hydrocarbons lead to a transformation not only of the composition of the feedstock, but also to a change in its physical and chemical properties and operational characteristics.

In order to identify the correlation between the component composition of the oil under study and its fragment composition, the study of hydrocarbons treated with electromagnetic by the action of NMR spectroscopy was carried out. To determine the fragmentary composition of oil by NMR spectroscopy, the ${ }^{1} \mathrm{H}$ and ${ }^{13} \mathrm{C}$ spectra of hydrocarbons were divided into a number of ranges of chemical shifts corresponding to different molecular fragments according to the accepted works [5]. This correlation of the fragment-structural characteristics of oil and petroleum products to the ranges of chemical shifts of the ${ }^{1} \mathrm{H}$ and ${ }^{13} \mathrm{C}$ NMR spectra is the most frequently used by researchers recently. The proton region of the chemical shifts of oil covers the range from 0.5 to $9.0 \mathrm{ppm}$ (Table 3 ), the considered carbon region of chemical shifts included from 0 to $193 \mathrm{ppm}$. Given that the sensitivity of proton spectra is 100 times greater than carbon, the greatest information in the NMR spectra of oil can be obtained from ${ }^{1} \mathrm{H}$ spectra.

Figure 2 shows the integrated sections of the ${ }^{1} \mathrm{H}$ NMR spectra of the initial oil (Figure 2a) and the oil after electromagnetic exposure (Figure 2b). By integrating the specified regions of the ${ }^{1} \mathrm{H}$ NMR spectra (Fig. 2) the fragmentary composition of the studied oil was determined (Table 1). The results obtained show that the composition of the oil samples under consideration contains terminal $\mathrm{CH}_{3}$ groups of long alkyl chains, which are manifested at a chemical shift value of $0.87 \mathrm{ppm}$. The presence of long alkyl chains of oil components gives the latter a high viscosity and bituminous consistency. In the initial oil and its samples, after exposure to electromagnetic radiation, there are no signals belonging to the range of 4.5-6.0 ppm. characterizing the presence of protons of an olefin nature. Therefore, these sections of the spectra in Fig. 2 are not integrated. In commercial oils, the content of olefin hydrocarbons usually does not exceed $1 \%$.

The content of aromatic protons according to the integral ${ }^{1} \mathrm{H}$ NMR intensities in the source oil does not exceed $2.1 \%$ during electromagnetic treatment.

The highest content of the fraction of Nu-type protons is observed in oil after electrohydraulic action. This is probably due to a slight break in the hydrocarbon chains and an increase in the terminal $\mathrm{CH}_{3}$ groups in the oil. At the same time, the viscosity of the oil should be reduced by reducing the length of the hydrocarbon chains. 

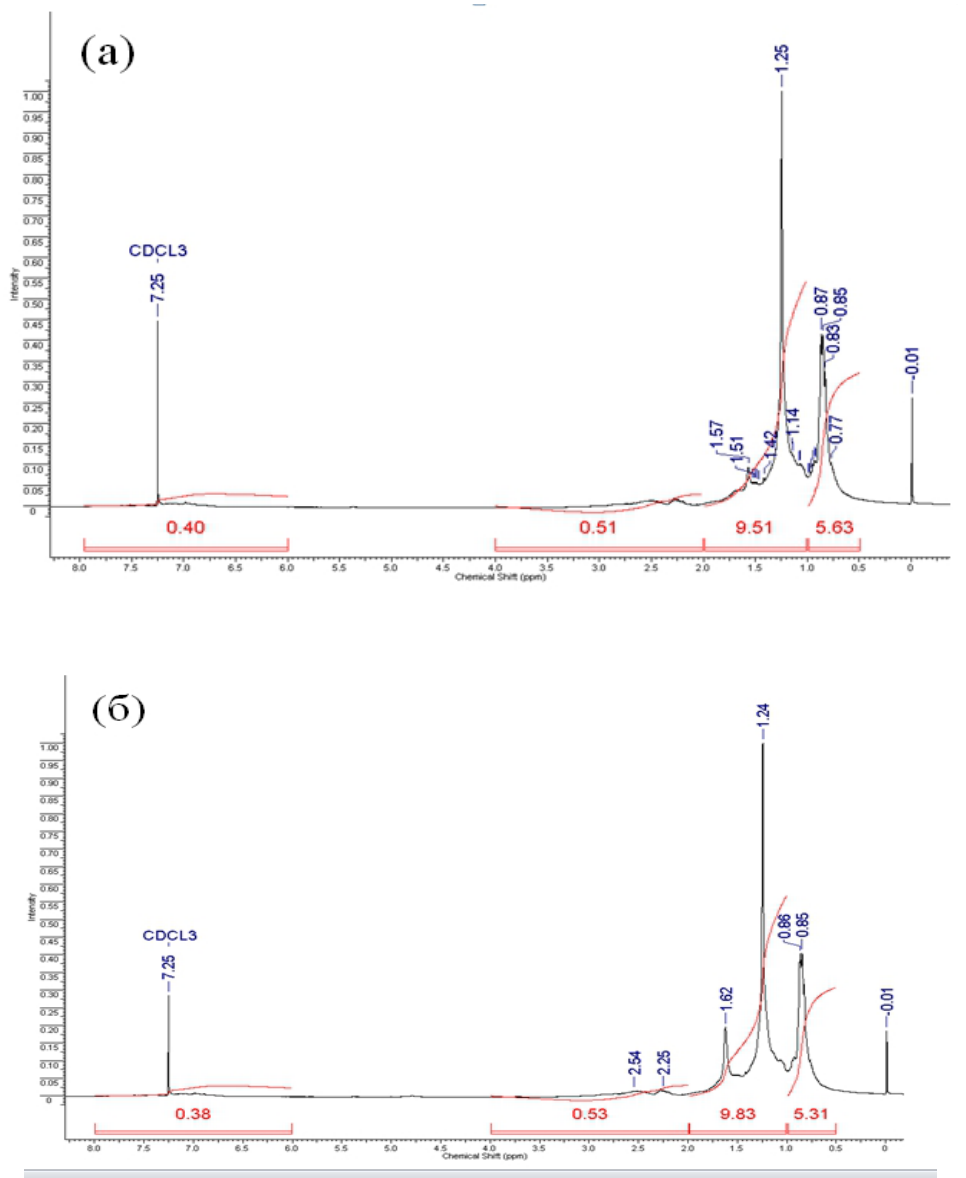

Fig.2. NMR spectra of initial fuel oil $\left({ }^{13} \mathrm{C}\right.$ spectrum $)$

Table 3. The ranges of chemical shifts of ${ }^{1} \mathrm{H}$ petroleum products NMR

\begin{tabular}{|c|c|c|}
\hline$\delta\left({ }^{1} \mathrm{H}\right), \mathrm{ppm}$ & $\begin{array}{c}\text { Atom } \\
\text { designation }\end{array}$ & Functional group \\
\hline $0.5-1.0$ & $\mathrm{H}_{\gamma}$ & $\begin{array}{r}\mathrm{CH}_{3} \text {-groups of saturated compounds. } \mathrm{CH}_{3} \text {-groups in } \\
\text { Y-and farther positions to the aromatic ring. }\end{array}$ \\
\hline $1.0-2.0$ & $\mathrm{H}_{\beta}$ & $\begin{array}{c}\mathrm{CH}_{2} \text { and } \mathrm{CH} \text { groups of saturated compounds. Hydrogen } \\
\text { atoms of } \beta \text {-methyl, } \beta \text {-and farther methylene and methine } \\
\text { groups at the aromatic ring }\end{array}$ \\
\hline $2.0-4.0$ & $\mathrm{H}_{\alpha}$ & $\begin{array}{r}\text { Hydrogen atoms in } \alpha \text {-position to aromatic and carbonyl } \\
\text { carbons, heteroatoms }\end{array}$ \\
\hline $4.5-6.3$ & $\mathrm{H}_{\text {ол }}$ & \begin{tabular}{c} 
Hydrogen atoms of olefin groups \\
\hline $6.3-9.0$
\end{tabular} \\
\hline
\end{tabular}




\section{Conclusions}

The results of the study presented in this paper showed the possibility of increasing the productivity of the gasoline fraction during electromagnetic oil treatment. It was shown that the increase in the yield of the open fraction in the zone of wave phenomena is accompanied by a significant change in the physical and chemical properties, the group composition of the resulting gasoline, opens up the possibility of using the direct distillation fraction obtained as a result of the activation of petroleum raw materials as a component of motor fuel for gasoline engines.

\section{References}

1. R.Kh. Salakhov, M. Seitzhanova, D.U. Bodykov, L.R. Sassykova, N.K. Zhakirova, T.M. Seilkhanov, Eurasian Chem-Technol. J. 22, 315 (2020)

2. L.R. Sassykova, N.K. Zhakirova, Y.A. Aubakirov et al., Rasayan J. Chem. 13,1444 (2020)

3. S. Fukase, F. Maruyama, J. Japan Pet. Inst. 37, 611 (1994)

4. R. Kh. Ibrasheva, V.S. Yemelyanova, L.R. Sassykova et al., Rasayan J. Chem. 13, 2370 (2020)

5. G.F. Pauli, B.U. Jaki, D.C. Lankin, J. Nat. Prod. 68, 133 (2005) 